\title{
Clinical features and short-term outcomes of cancer patients with suspected and unsuspected pulmonary embolism: the EPIPHANY study
}

\author{
Carme Font ${ }^{1}$, Alberto Carmona-Bayonas ${ }^{2}$, Carmen Beato ${ }^{3}$, Òscar Reig $^{1}$, \\ Antonia Sáez ${ }^{4}$, Paula Jiménez-Fonseca ${ }^{5}$, Juana M. Plasencia ${ }^{6}$, \\ David Calvo-Temprano ${ }^{7}$, Marcelo Sanchez ${ }^{8}$, Mariana Benegas $^{8}$, \\ Mercedes Biosca 9 , Diego Varona ${ }^{10}$, Maria Angeles Vicente ${ }^{2}$, Laura Faez ${ }^{5}$, \\ Maria del Pilar Solís ${ }^{5}$, Irma de la Haba ${ }^{11}$, Maite Antonio ${ }^{11}$, Olga Madridano ${ }^{12}$, \\ Eduardo Castañon ${ }^{13}$, María Jose Martinez ${ }^{14}$, Pablo Marchena ${ }^{15}$, \\ Avinash Ramchandani ${ }^{16}$, Angel Dominguez ${ }^{17}$, Alejandro Puerta ${ }^{18}$, \\ David Martinez de la Haza ${ }^{19}$, Jesus Pueyo ${ }^{20}$, Susana Hernandez ${ }^{21}$, \\ Angela Fernandez-Plaza ${ }^{22}$, Lourdes Martinez-Encarnacion ${ }^{22}$, Mar Martin ${ }^{12}$, \\ Gema Marin ${ }^{23}$, Francisco Ayala ${ }^{2}$, Vicente Vicente ${ }^{2}$ and Remedios Otero ${ }^{24}$ on \\ behalf of the Asociación para la Investigación de la Enfermedad \\ Tromboembólica de la región de Murcia
}

@ERSpublications

Predictors of 30-day mortality in cancer patients with suspected and unsuspected pulmonary embolism http://ow.ly/Nu0k305t5KD

Cite this article as: Font C, Carmona-Bayonas A, Beato C, et al. Clinical features and short-term outcomes of cancer patients with suspected and unsuspected pulmonary embolism: the EPIPHANY study. Eur Respir J 2017; 49: 1600282 [https://doi.org/10.1183/13993003.00282-2016].

ABSTRACT The study aimed to identify predictors of overall 30-day mortality in cancer patients with pulmonary embolism including suspected pulmonary embolism (SPE) and unsuspected pulmonary embolism (UPE) events. Secondary outcomes included 30- and 90-day major bleeding and venous thromboembolism (VTE) recurrence.

The study cohort included 1033 consecutive patients with pulmonary embolism from the multicentre observational ambispective EPIPHANY study (March 2006-October 2014). A subgroup of 497 patients prospectively assessed for the study were subclassified into three work-up scenarios (SPE, truly asymptomatic UPE and UPE with symptoms) to assess outcomes.

The overall 30 -day mortality rate was $14 \%$. The following variables were associated with the overall 30 day mortality on multivariate analysis: VTE history, upper gastrointestinal cancers, metastatic disease, cancer progression, performance status, arterial hypotension $<100 \mathrm{mmHg}$, heart rate $>110$ beats. $\mathrm{min}^{-1}$, basal oxygen saturation $<90 \%$ and SPE (versus overall UPE).

The overall 30-day mortality was significantly lower in patients with truly asymptomatic UPE events (3\%) compared with those with UPE-S $(20 \%)$ and SPE $(21 \%)(\mathrm{p}<0.0001)$. Thirty- and 90-day VTE recurrence and major bleeding rates were similar in all the groups.

In conclusion, variables associated with the severity of cancer and pulmonary embolism were associated with short-term mortality. Our findings may help to develop pulmonary embolism risk-assessment models in this setting. 
Affiliations: ${ }^{1}$ Medical Oncology Dept, IDIBAPS/Translational Genomics and Targeted Therapeutics in Solid Tumors, Hospital Clinic de Barcelona, Barcelona, Spain. ${ }^{2}$ Medical Oncology and Hematology Dept, Hospital Universitario Morales Meseguer, Murcia, Spain. ${ }^{3}$ Medical Oncology Dept, Hospital Nisa-Aljarafe, Sevilla, Spain. ${ }^{4}$ Statistical Dept, Axioma Comunicaciones, Sevilla, Spain. ${ }^{5}$ Medical Oncology Dept, Hospital Universitario Central de Asturias, Oviedo, Spain. ${ }^{6}$ Radiology Dept, Hospital Universitario Morales Meseguer, Murcia, Spain. ${ }^{7}$ Radiology Dept, Hospital Universitario Central de Asturias, Oviedo, Spain. ${ }^{8}$ Radiodiagnostic Dept, Hospital Clinic de Barcelona, Barcelona, Spain. ${ }^{9}$ Medical Oncology Dept, Hospital Universitari Vall d'Hebron, Barcelona, Spain. ${ }^{10}$ Radiology Dept, Hospital Universitari Vall d'Hebron, Barcelona, Spain. ${ }^{11}$ Medical Oncology Dept, Hospital Duran i Reynals, Institut Catalàd'Oncologia, Hospitalet de Llobregat, Barcelona, Spain. ${ }^{12}$ Internal Medicine Dept, Hospital Infanta Sofía. San Sebastian de los Reves, Madrid, Spain. ${ }^{13}$ Medical Oncology Dept, Clinica Universidad de Navarra, Pamplona, Spain. ${ }^{14}$ Medical Oncology Dept, Hospital Santa Lucía, Cartagena, Murcia, Spain. ${ }^{15}$ Internal Medicine Dept, Hospital Sant Joan de Deu, Esplugues de Llobregat, Barcelona, Spain. ${ }^{16}$ Medical Oncology Dept, Hospital Insular de Gran Canaria, Las Palmas, Spain. ${ }^{17}$ Radiology Dept, Hospital Nisa-Aljarafe, Sevilla, Spain. ${ }^{18}$ Radiology Dept, Hospital Reina Sofía, Murcia, Spain. ${ }^{19}$ Radiology Dept, Hospital Duran i Reynals, InstitutCatalàd'Oncologia, Hospitalet de Llobregat, Barcelona, Spain. ${ }^{20}$ Radiology Dept, Clinica Universidad de Navarra, Pamplona, Spain. ${ }^{21}$ Radiology Dept, Hospital Infanta Sofía. San Sebastian de los Reves, Madrid, Spain. ${ }^{22}$ Radiology Dept, Hospital Santa Lucía, Cartagena, Murcia, Spain. ${ }^{23}$ Medical Oncology Dept, Hospital Clínico Universitario Virgen de la Arrixaca, Murcia, Spain. ${ }^{24}$ Dept of Pneumology, Hospital Universitario Virgen del Rocío (HUVR-IBiS). CIBERES, Sevilla, Spain.

Correspondence: Carme Font, Medical Oncology Dept, IDIBAPS/Translational Genomics and Targeted Therapeutics in Solid Tumors, Hospital Clinic de Barcelona, Villarroel 170, 08036-Barcelona, Spain.

E-mail: CFONTAclinic.ub.es

\section{Introduction}

Venous thromboembolism (VTE) including deep vein thrombosis and pulmonary embolism is associated with increased mortality in cancer patients [1]. In turn, cancer has been found to be an independent predictor of death in series of patients with acute pulmonary embolism [2,3] and has been included as a predictive variable in validated pulmonary embolism prognostic models [4-6]. Thus, it implies that it is practically impossible for cancer patients to be classified in the "low risk", categories which limits their utility in this setting. This fact has led to the development of cancer-specific risk-assessment tools for acute symptomatic or suspected pulmonary embolism (SPE) [7-9].

However, incidental or unsuspected pulmonary embolism (UPE) is reported in 1-5\% of scheduled computed tomography (CT) scans performed in cancer patients for reasons other than pulmonary embolism suspicion [10], representing about half of the pulmonary embolisms currently diagnosed in oncology [11-14]. Data from retrospective and observational studies suggest that the embolic burden in UPE is similar to that in SPE $[12,14,15]$, and that UPE could have an adverse impact on patient survival [16]. In addition, similar outcomes have been observed in overall mortality, major bleeding and recurrent VTE on comparing patients with UPE to those with symptomatic events [14, 17]. Thus, in the absence of data from controlled trials, international clinical guidelines currently recommend that incidental VTE be treated similarly to symptomatic thrombosis [18-20].

Nonetheless, limited information is available about the specific predictors of adverse outcomes in patients with cancer-associated pulmonary embolism, particularly in cases of UPE. We hypothesised that variables related to the clinical scenario at pulmonary embolism presentation such as inpatient versus outpatient setting, cancer type and severity as well as the characteristics of the thrombotic event itself (pulmonary embolism suspicion, abnormality in vital signs) might influence the short-term mortality of these patients. Therefore, the aim of the present study was to assess the variables associated with 30-day mortality in cancer patients with pulmonary embolism (SPE and UPE events), as potentially useful to develop risk-classification tools for individualising acute-phase pulmonary embolism management in this setting. We also assessed the outcomes according to three work-up scenarios: SPE, UPE with symptoms (UPE-S) and truly asymptomatic UPE (TA-UPE) events.

\section{Methods}

Study design

This multicentre observational ambispective EPIPHANY study was aimed to describe the clinical characteristics and outcomes of patients with cancer-associated pulmonary embolism including acute suspected

This article has supplementary material available from erj.ersjournals.com

Received: Feb 062016 | Accepted after revision: Oct 132016

LEO Pharma Spain provided funding for the study via an academic grant. Funding information for this article has been deposited with the Open Funder Registry.

Conflict of interest: None declared. 
and unsuspected events [21]. The primary endpoint was the overall 30-day mortality rate and the identification of variables associated with death. Secondary outcomes included: 1) 30- and 90-day major bleeding and VTE recurrence rates; and 2) Outcome measures according to three clinical pulmonary embolism work-up scenarios (SPE, UPE-S and TA-UPE) in patients prospectively assessed for the present study (table 1).

The study was conducted in 14 Spanish teaching hospitals. A clinician experienced in supportive cancer care gathered and updated clinical information and a senior radiologist reviewed the pulmonary embolism-related radiological data at each participating centre. A centralised web platform specifically designed for the study was used for electronic data collection. The inclusion of patients started on October 1, 2013 and ended on October 31, 2014. Each patient was included in the study only once with only one "index pulmonary embolism" evaluable for acute variables. If multiple pulmonary embolisms occurred in the same patient, they were considered as medical history (if occurring $>3$ months prior to the index pulmonary embolism) or as VTE recurrence during follow-up.

A minimum observation period of 90 days after the index pulmonary embolism was required. On-site or remote monitoring was performed at all sites for the adjudication of the critical outcome measures. Bleeding events were classified as major according to the International Society of Thrombosis and Haemostasis criteria [24]. Recurrent VTE diagnosis during follow-up required objective confirmation. Causes of death were recorded according to a multiple-choice classification: pulmonary embolism, cancer progression, bleeding and/or infection.

\section{Study population}

The study was carried out with ambispective recruitment of consecutively eligible patients including: 1) welldocumented retrospectively reviewed cases according to a systematic search of local radiology or clinical files from March 2006 onwards up to October 2013 partially reported as part of previous studies [12, 25]; and 2) patients prospectively assessed according to local protocols specifically developed for the present study aimed to ensure the inclusion of all new cancer-associated pulmonary embolism cases in each participating centre after October 2013.

The inclusion criteria were: i) adult (>18 years); ii) active cancer or receiving adjuvant chemotherapy; iii) objectively confirmed pulmonary embolism including SPE and UPE events; and iv) anticoagulation as part of daily clinical practice according to international guidelines [18-20,26].

TABLE 1 Work-up scenarios defined for clinical cancer-associated pulmonary embolism classification

Suspected pulmonary

embolism
Unsuspected pulmonary embolism

\section{With symptoms}

No

Inpatient or outpatient

Unscheduled imaging test specifically performed to depict pulmonary embolism

\section{Confirmatory imaging test}

Clinical findings at pulmonary embolism presentation
Inpatient or outpatient

Either scheduled or unplanned conventional computed tomography scan performed for reasons other than pulmonary embolism suspicion

Conventional computed tomography scan

Presence of new symptoms and/or abnormality in vital signs potentially attributable to pulmonary embolism
CTPA or

high probability ventilation/perfusion pulmonary scintigraphy according to the PIOPED criteria [22]

Presence of symptoms and/or abnormality in vital signs that led clinicians to depict pulmonary embolism

\section{Truly asymptomatic}

No

Outpatient

Scheduled computed tomography scan performed for reasons other than pulmonary embolism suspicion, usually cancer monitoring

Conventional computed tomography scan
No symptoms suspicious of pulmonary embolism and vital signs within normal limits [23] including: blood pressure $\geqslant 100 \mathrm{mmHg}$, heart rate $<100$ beats $\cdot \mathrm{min}^{-1}$ and basal oxygen saturation $\geqslant 95 \%$ 
The exclusion criteria were: i) pulmonary embolism more than 1 month prior to cancer diagnosis; ii) pulmonary embolism more than 4 weeks after completing adjuvant chemotherapy; and iii) patients receiving other adjuvant therapies such as hormone therapy after radical anticancer treatments.

The study was approved by the local Clinical Research Ethics Boards at each participating hospital. Informed written consent was obtained from all the prospectively enrolled participants.

\section{Clinical variables}

The demographic and clinical variables at pulmonary embolism diagnosis included age, sex, VTE history, chronic cardiopulmonary conditions, body weight, inpatient or ambulatory, performance status according to the Eastern Cooperative Oncology Group (ECOG) scale [27], cancer type and stage, cancer status according to standard oncological evaluation (complete or partial response, stable disease or cancer progression) [28], anticancer therapies within 1 month before pulmonary embolism, vital signs (blood pressure and heart rate), basal pulse-oximetry, presence of symptoms (yes or no) as a general category, the presence and characteristics of dyspnoea (sudden, progressive or self-limited), chest pain, syncope or faint and haemoptysis. We also recorded the attribution of symptoms as a multiple-choice option (pulmonary embolism, cancer, chemotherapy and/or other comorbidities) and the initial (first 5-10 days) and long-term (beyond the initial treatment up to 3 months) pulmonary embolism management.

\section{Pulmonary embolism diagnosis and classification}

All pulmonary embolisms were objectively confirmed by standard radiological methods. The radiological variables analysed for the present study were categorised as: 1) single versus multiple pulmonary embolism; 2) unilateral versus bilateral lung involvement; and 3) troncular (principal arteries), lobar, segmentary, multi-subsegmentary and isolated subsegmentary pulmonary embolism according to the most proximal clot seen in pulmonary arteries.

Table 1 summarises the criteria used for pulmonary embolism classification based on the intention of clinicians when ordering imaging tests. Briefly, SPE was diagnosed when confirmatory imaging tests (ventilation/perfusion scintigraphy or computed tomography with pulmonary angiography (CTPA)) were specifically performed to depict pulmonary embolism whereas UPE when diagnosed on conventional computed tomography scans performed for reasons other than pulmonary embolism suspicion.

Based on additional clinical parameters, patients with UPE were subclassified as UPE-S versus TA-UPE, this latter subgroup defined as outpatient undergoing a scheduled computed tomography scan and without symptoms or signs suspicious of pulmonary embolism at clinical evaluation.

\section{Statistical analysis}

Categorical variables were compared by the Chi-squared or Fisher's test and continuous variables were compared using the t-test or Mann-Whitney U-test as appropriate according to normality and homoscedasticity. Univariate and multivariate logistic regression analyses were performed in the whole cohort to determine the strength of association between each potential predictor and overall 30-day mortality. We included variables related to demographics, comorbidities, cancer-related characteristics, radiological findings, vital signs and the variable SPE (versus UPE) as a surrogate of having symptoms in the whole cohort to compare patients who died or were alive at 30 days after the index pulmonary embolism. Variables with p-values of $<0.10$ on univariate analysis were eligible for multivariate analyses using the backward conditional method. Overall survival at 90 days of follow-up was estimated with the Kaplan-Meier method and comparisons between the work-up scenarios classification groups were made using the log-rank test. The overall survival was calculated as the time from the index pulmonary embolism to death or censored data at 90 days of follow-up. Statistical significance was established at $\mathrm{p}<0.05$ (two-sided). Calculations were carried out with the IBM SPSS Statistics 22 software (SPSS Inc., Chicago, IL, USA).

\section{Results}

\section{Patient characteristics}

We enrolled 1033 consecutive eligible patients (429 with SPE and 604 UPE) with prospective assessment of clinical data in 497 cases (48\% of the whole cohort) (figure 1).

Patients with SPE were diagnosed by CTPA in 402 cases and by lung scintigraphy in 27 cases. The UPE group comprised: 557 stable patients who underwent scheduled computed tomography scans, with 43 cases undergoing an unplanned conventional computed tomography scan for clinical suspicion of cancer progression, and in four additional patients doubtful pulmonary embolism images found in the scheduled computed tomography scan required imaging confirmation by CTPA. 


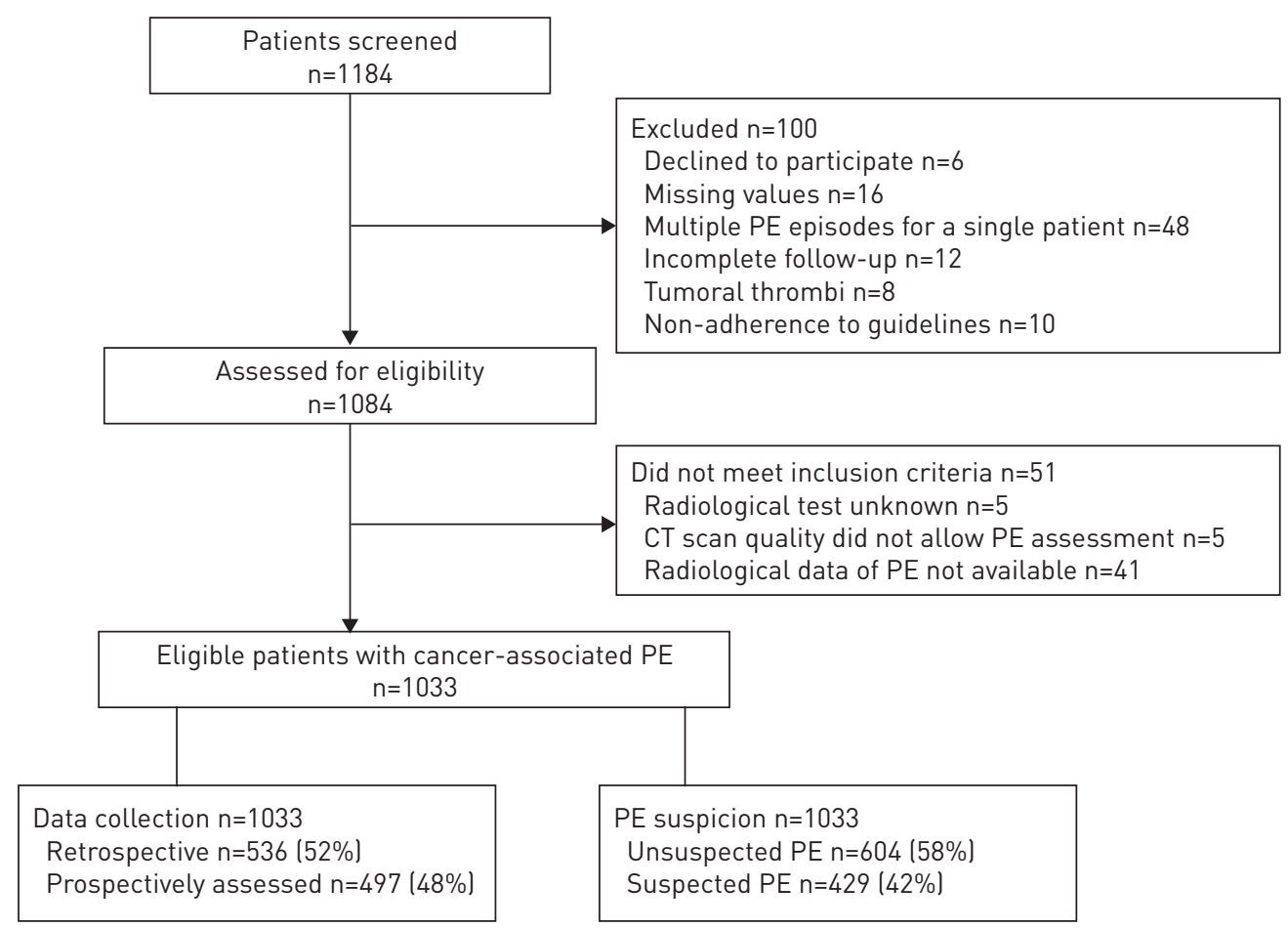

FIGURE 1 EPIPHANY study flowchart diagram. PE: pulmonary embolism; CT: computed tomography.

On comparing the baseline characteristics of patients with UPE and SPE (table 2) we found that those with SPE were more likely to have concomitant VTE, chronic lung and heart conditions and major surgery within 30 days prior to the pulmonary embolism compared to patients with UPE. In turn, colorectal and upper gastrointestinal cancers, metastatic disease, good performance status ECOG 0-1 and biological anticancer therapies were more frequently observed in patients with UPE.

We also compared the main clinical variables of patients enrolled in the study according to prospective and retrospective data collection (supplementary material). It is of note that no differences were found with regard to most of the variables recorded although significant differences were observed in the following variables: concomitant VTE and being inpatient at pulmonary embolism diagnosis (more frequently observed in the retrospective group) and chronic lung condition, lung cancer, metastatic disease, presence of symptoms and chest pain, the latter being more likely found in the prospectively assessed group.

The characteristics of 497 prospectively assessed patients according to triple clinical scenario classification (31\% TA-UPE, 26\% UPE-S and 43\% SPE) are shown in table 3. Patients with UPE-S were more likely to have metastatic disease and cancer progression compared to patients with TA-UPE and SPE. In turn, good performance status (ECOG 0-1), chemotherapy and biological therapies were more frequent in patients with TA-UPE whereas greater abnormality in vital signs and lower oxygen saturation were more frequent in patients with SPE.

Of note, overall, a remarkable $44 \%$ of patients did not complain of symptoms including: $100 \%$ of patients with TA-UPE; 36\% of cases with UPE-S who fulfilled this category for abnormal vital signs and/or for being inpatients for other reasons at pulmonary embolism diagnosis and $8 \%$ of patients with SPE in whom clinicians decided to depict pulmonary embolism based on abnormal vital signs and/or low oxygen saturation.

With regard to the radiological variables analysed, patients with SPE were more likely to have bilateral and multiple pulmonary embolisms as well as involvement of troncular arteries compared to patients with unsuspected events. It was of note that subsegmentary arteries as the most proximal vascular involvement accounted for less than $5 \%$ of the cases in all the groups.

Overall 30-day mortality and predictors of death

144 patients died during the first 30 days after the index event (14\% of the whole cohort). The following variables were associated with death at the 30-day of follow-up on multivariate analysis (table 4): VTE history, upper gastrointestinal cancers, metastatic disease, cancer progression, performance status ECOG $>2$, blood pressure $<100 \mathrm{mmHg}$, heart rate $>110$ beats $\cdot \mathrm{min}^{-1}$, basal oxygen saturation $<90 \%$ and SPE 
TABLE 2 Baseline characteristics of the whole cohort and according to the presence of UPE or SPE

\begin{tabular}{|c|c|c|c|c|}
\hline & Overall & UPE & SPE & p-value \\
\hline Total $\mathbf{n}$ & 1033 & 604 & 429 & \\
\hline \multicolumn{5}{|l|}{ Age } \\
\hline$\leqslant 60$ years & 340 (33) & 195 (32) & 145 (34) & NS \\
\hline $61-70$ years & 329 (32) & 202 (33) & $127(30)$ & \\
\hline $71-80$ years & $285(27)$ & $167(27)$ & $118(27)$ & \\
\hline$\geqslant 80$ years & $79(8)$ & $40(7)$ & 39 (9) & \\
\hline Age years & $65 \pm 11.5$ & $65 \pm 11.4$ & $65 \pm 12.5$ & NS \\
\hline Male & $561(54)$ & 333 (55) & 228 (53) & NS \\
\hline Weight kg & $72.0 \pm 14.4$ & $71.7 \pm 14.0$ & $72.4 \pm 14.9$ & NS \\
\hline VTE history & 120 (12) & $67(11)$ & $53(12)$ & NS \\
\hline Concomitant VTE & $260(25)$ & $133(22)$ & $127(30)$ & 0.004 \\
\hline Inpatient at pulmonary embolism diagnosis & $236(23)$ & $132(22)$ & $104(24)$ & NS \\
\hline Chronic lung disease & $125(12)$ & $60(10)$ & $65(15)$ & 0.008 \\
\hline Chronic heart disease & $56(5)$ & $26(4)$ & $30(7)$ & 0.042 \\
\hline \multicolumn{5}{|l|}{ Tumour type } \\
\hline Lung & $265(26)$ & $152(25)$ & $113(26)$ & NS \\
\hline Colorrectal & $185(18)$ & $121(20)$ & $64(15)$ & 0.021 \\
\hline Upper gastrointestinal & $144(14)$ & $94(16)$ & $50(12)$ & 0.044 \\
\hline Breast & $119(12)$ & $64(11)$ & $55(13)$ & NS \\
\hline Urological & $94(9)$ & $49(8)$ & $45(11)$ & NS \\
\hline Gynaecological & $79(8)$ & $44(7)$ & $35(8)$ & NS \\
\hline Other & $147(14)$ & $80(13)$ & $67(16)$ & NS \\
\hline Tumour stage & & & & 0.004 \\
\hline I & $37(4)$ & $16(3)$ & $21(5)$ & \\
\hline II & $64(6)$ & $29(5)$ & $35(8)$ & \\
\hline III & $169(16)$ & $89(15)$ & 80 (19) & \\
\hline IV & $763(74)$ & $470(78)$ & $293(68)$ & \\
\hline Cancer progression & 391 (38) & 229 (38) & $162(38)$ & NS \\
\hline ECOG & & & & $<0.001$ \\
\hline ECOG 0-1 & $554(54)$ & 359 (59) & $195(45)$ & \\
\hline ECOG 2 & $290(28)$ & 149 (25) & 141 (33) & \\
\hline ECOG 3 & $179(17)$ & $89(15)$ & $90(21)$ & \\
\hline ECOG 4 & $10(1)$ & $7(1)$ & $3(1)$ & \\
\hline \multicolumn{5}{|l|}{ Therapy 30 days prior to VTE } \\
\hline Chemotherapy & $556(54)$ & $338(56)$ & $218(51)$ & NS \\
\hline Cisplatin-based & $111(11)$ & $69(11)$ & $42(10)$ & NS \\
\hline Biological therapy & $138(13)$ & $102(17)$ & $36(8)$ & $<0.0001$ \\
\hline Hormone therapy & $87(8)$ & $45(7)$ & $42(9)$ & NS \\
\hline Major surgery & $73(7)$ & $27(4)$ & $46(11)$ & $<0.0001$ \\
\hline ESA & $47(4)$ & $29(5)$ & $18(4)$ & NS \\
\hline
\end{tabular}

Data are presented as $\mathrm{n}(\%)$ or mean $\pm \mathrm{SD}$, unless otherwise stated. UPE: unsuspected pulmonary embolism; SPE: suspected pulmonary embolism; VTE: venous thromboembolism; ECOG: Eastern Cooperative Oncology Group; ESA: Erythropoietin stimulating agent; NS: Not significant.

(versus overall UPE). Notably, neither age as a continuous or categorised variable nor chronic lung and heart conditions were associated with 30-day mortality in our study.

It was of note that the 30-day mortality was increased when the pulmonary embolism occurred in inpatients compared to pulmonary embolism in ambulatory patients, although the difference did not reach statistical differences on multivariate analysis $(\mathrm{p}=0.064)$.

Pulmonary embolism management and outcomes according to pulmonary embolism work-up scenarios

Table 5 shows the information about the initial and long-term pulmonary embolism management as well as the 30- and 90-day outcomes in the whole cohort and according to the pulmonary embolism work-up scenarios. The overall 30-day mortality rate was significantly lower in patients with TA-UPE compared to those with UPE-S and SPE (3\%, 20\% and 21\%, respectively; $\mathrm{p}<0.001)$.

With regard to the causes of death at the 30-day follow-up, clinicians considered the pulmonary embolism as a single cause of 30 -day death in $2 \%$ of patients (19 out of 1033 considering the whole cohort and nine out of 


\begin{tabular}{|c|c|c|c|c|c|}
\hline & Overall & TA-UPE & UPE-S & SPE & p-value \\
\hline Total $\mathbf{n}$ & 497 & $154(31)$ & 12926 & $214(43)$ & \\
\hline Age years & & & & & NS \\
\hline$\leqslant 60$ & $168(34)$ & $52(34)$ & $46(35)$ & 70 (33) & \\
\hline $61-70$ & $163(33)$ & $51(33)$ & $41(32)$ & $71(33)$ & \\
\hline $71-80$ & 135 (27) & $40(26)$ & $36(28)$ & $59(28)$ & \\
\hline$>80$ & $31(6)$ & $11(7)$ & $6(5)$ & $14(6)$ & \\
\hline Male & 283 (57) & $90(58)$ & $72(56)$ & 121 (57) & NS \\
\hline Chronic lung disease & 75 (15) & $17(11)$ & $18(14)$ & 40 (19) & NS \\
\hline Chronic heart disease & $28(6)$ & $5(3)$ & $6(5)$ & 17 (8) & NS \\
\hline VTE history & $60(12)$ & $16(10)$ & $15(12)$ & $29(14)$ & NS \\
\hline Concomitant DVT & $107(22)$ & $28(18)$ & $27(21)$ & $52(24)$ & NS \\
\hline Inpatient at pulmonary embolism diagnosis & $100(20)$ & 0 & $53(41)$ & $47(22)$ & $<0.001$ \\
\hline Tumour type & & & & & NS \\
\hline Lung & $153(31)$ & $41(27)$ & $45(35)$ & $67(31)$ & \\
\hline Colorrectal & 78 (15) & $32(21)$ & $15(12)$ & $31(14)$ & \\
\hline Upper gastrointestinal & 64 (13) & $22(14)$ & $18(14)$ & $24(11)$ & \\
\hline Breast & $53(11)$ & $14(9)$ & $12(9)$ & $27(13)$ & \\
\hline Urological & $48(10)$ & $17(11)$ & $12(9)$ & $19(9)$ & \\
\hline Gynaecological & $41(8)$ & $14(9)$ & $10(8)$ & $17(8)$ & \\
\hline Other & 60 (12) & $14(9)$ & $17(13)$ & $29(14)$ & \\
\hline Metastatic cancer & $391(79)$ & $124(80)$ & $111(86)$ & $156(73)$ & 0.016 \\
\hline Cancer progression (yes) & $198(40)$ & $54(35)$ & $62(48)$ & $82(38)$ & 0.07 \\
\hline ECOG 0-1 & $263(53)$ & $109(71)$ & $55(43)$ & $99(46)$ & $<0.001$ \\
\hline \multicolumn{6}{|l|}{ Treatments 30 days prior pulmonary embolism } \\
\hline Major surgery & $38(8)$ & $7(5)$ & $3(2)$ & $28(13)$ & $<0.001$ \\
\hline Chemotherapy & $272(55)$ & $98(64)$ & $56(43)$ & $118(55)$ & 0.003 \\
\hline Biological & 72 (15) & $35(23)$ & $15(12)$ & $22(10)$ & 0.002 \\
\hline Hormonal & $39(8)$ & $14(9)$ & $7(5)$ & $18(8)$ & NS \\
\hline No symptoms & $217(44)$ & $154(100)$ & $47(36)$ & $16(8)$ & $<0.001$ \\
\hline Dyspnoea & & & & & $<0.001$ \\
\hline No & $247(50)$ & $154(100)$ & $59(47)$ & $34(16)$ & \\
\hline Sudden & 92 (19) & 0 & $11(9)$ & $81(38)$ & \\
\hline Progressive & $143(29)$ & 0 & $48(38)$ & $95(44)$ & \\
\hline Self-limited & $12(2)$ & 0 & $8(6)$ & $4(2)$ & \\
\hline Chest pain & $99(20)$ & 0 & $24(19)$ & 75 (35) & $<0.001$ \\
\hline Syncope or faint & $28(6)$ & 0 & $7(5)$ & $21(10)$ & $<0.001$ \\
\hline Haemoptysis & $18(4)$ & 0 & $7(5)$ & $11(5)$ & 0.015 \\
\hline \multicolumn{6}{|l|}{ Attribution of symptoms } \\
\hline PE & $263(53)$ & 0 & $79(61)$ & $184(86)$ & $<0.001$ \\
\hline Cancer & 86 (17) & 0 & $36(28)$ & $50(23)$ & $<0.001$ \\
\hline Other conditions & 47 (10) & 0 & $14(11)$ & $33(15)$ & $<0.001$ \\
\hline Heart rate $>100$ beats $\cdot \mathrm{min}^{-1}$ & $169(34)$ & 0 & $39(31)$ & $129(60)$ & $<0.001$ \\
\hline Blood pressure $<100 \mathrm{mmHg}$ & $47(9)$ & 0 & $14(11)$ & 33 (15) & $<0.001$ \\
\hline Oxygen saturation & & & & & $<0.001$ \\
\hline$\geqslant 95 \%$ & $341(68)$ & $154(100)$ & 94 (73) & $93(43)$ & \\
\hline $91-94 \%$ & $78(16)$ & 0 & $24(18)$ & $54(25)$ & \\
\hline $81-90 \%$ & $63(13)$ & 0 & $10(8)$ & $53(25)$ & \\
\hline$<80$ & $15(3)$ & 0 & $1(1)$ & $14(7)$ & \\
\hline \multicolumn{6}{|l|}{ Radiological findings } \\
\hline Bilateral lung involvement & $264(53)$ & $67(43)$ & $62(48)$ & 135 (63) & $<0.001$ \\
\hline Multiple pulmonary embolism & 409 (82) & $117(76)$ & $106(82)$ & 186 (87) & 0.025 \\
\hline \multicolumn{6}{|l|}{ Most proximal clot seen: } \\
\hline Troncular & $154(31)$ & $35(23)$ & $38(30)$ & 81 (39) & 0.005 \\
\hline Lobar & $153(31)$ & $58(38)$ & $47(37)$ & $48(23)$ & 0.003 \\
\hline Segmentary & $136(28)$ & $50(33)$ & $26(20)$ & $60(29)$ & 0.076 \\
\hline Multi-sub-segmentary & $12(3)$ & $3(2)$ & $2(2)$ & $7(3.5)$ & NS \\
\hline Isolated subsegmentary & $4(1)$ & $1(1)$ & 0 & $3(1)$ & NS \\
\hline
\end{tabular}

Data are presented as $n(\%)$, unless otherwise stated. TA-UPE: truly asymptomatic unsuspected pulmonary embolism; UPE-S: unsuspected pulmonary embolism with symptoms; SPE: suspected pulmonary embolism; VTE: venous thromboembolism; DVT: deep vein thrombosis; ECOG: Eastern Cooperative Cancer Group; NS: not significant. 
TABLE 4 Univariate and multivariate analysis of covariates associated with the overall 30-day mortality in the whole cohort

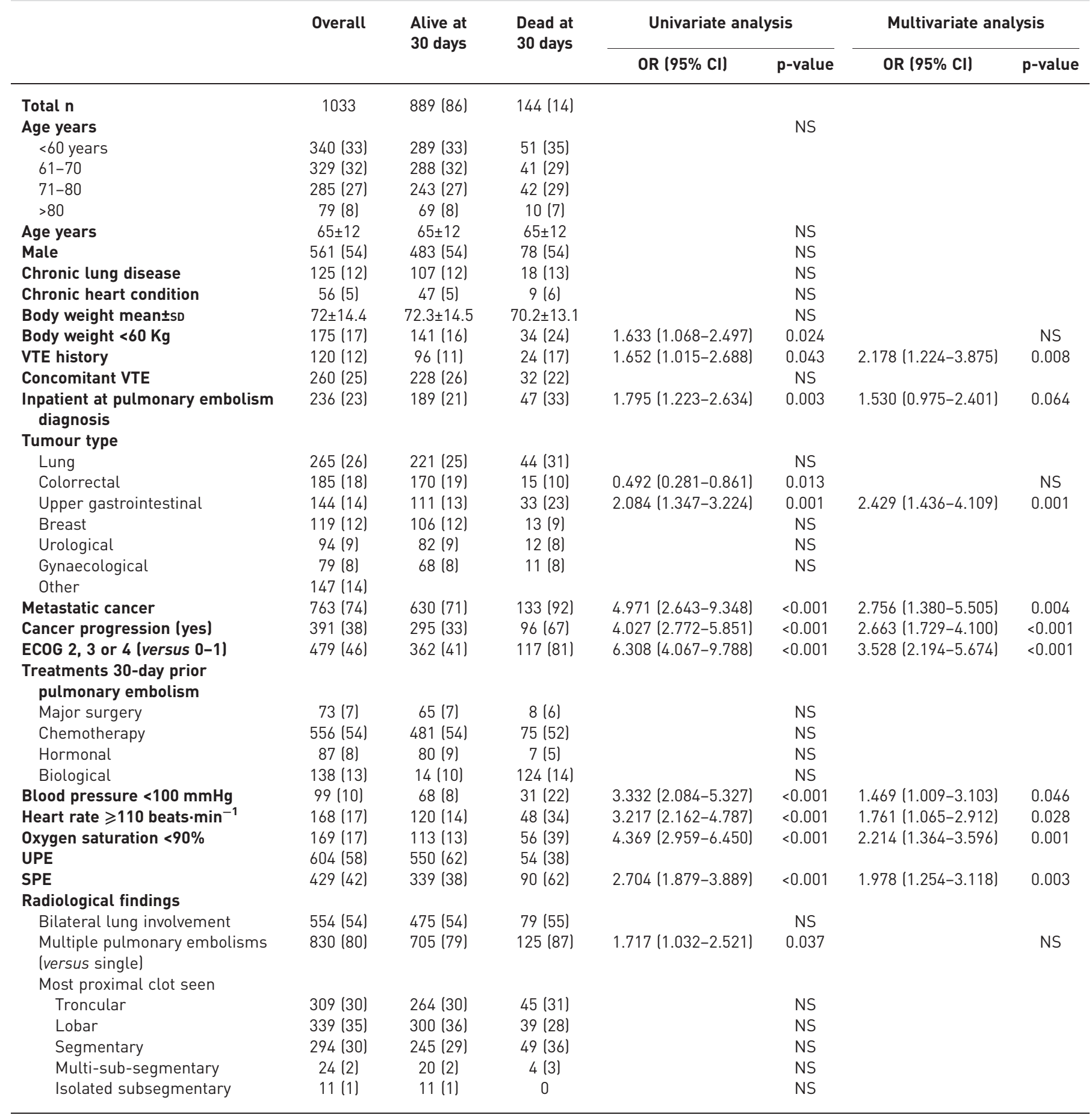

Data are presented as $\mathrm{n}(\%)$ or mean \pm SD, unless otherwise stated. VTE: venous thromboembolism; ECOG: Eastern Cooperative Cancer Group; UPE: unsuspected pulmonary embolism; SPE: suspected pulmonary embolism; NS: not significant.

497 in prospectively assessed patients). Notably, death was more frequently related to pulmonary embolism (including pulmonary embolism as a single cause and pulmonary embolism in association with other conditions) in patients with SPE while death was mainly attributed to cancer in patients with UPE events.

The overall mortality rates at the 90 -day follow-up were $27 \%$ considering the whole cohort and $29 \%$ in the overall prospectively assessed cases. At 90 days, the mortality rates were significantly higher in patients with UPE-S (43\%) than in those with SPE $(33 \%)$ and TA-UPE $(12 \%)(\mathrm{p}<0.001)$, and cancer was 
TABLE 5 Pulmonary embolism management and outcomes in the whole cohort ( $n=1033$ ) and the triple work-up scenarios ( $\mathrm{n}=497$ )

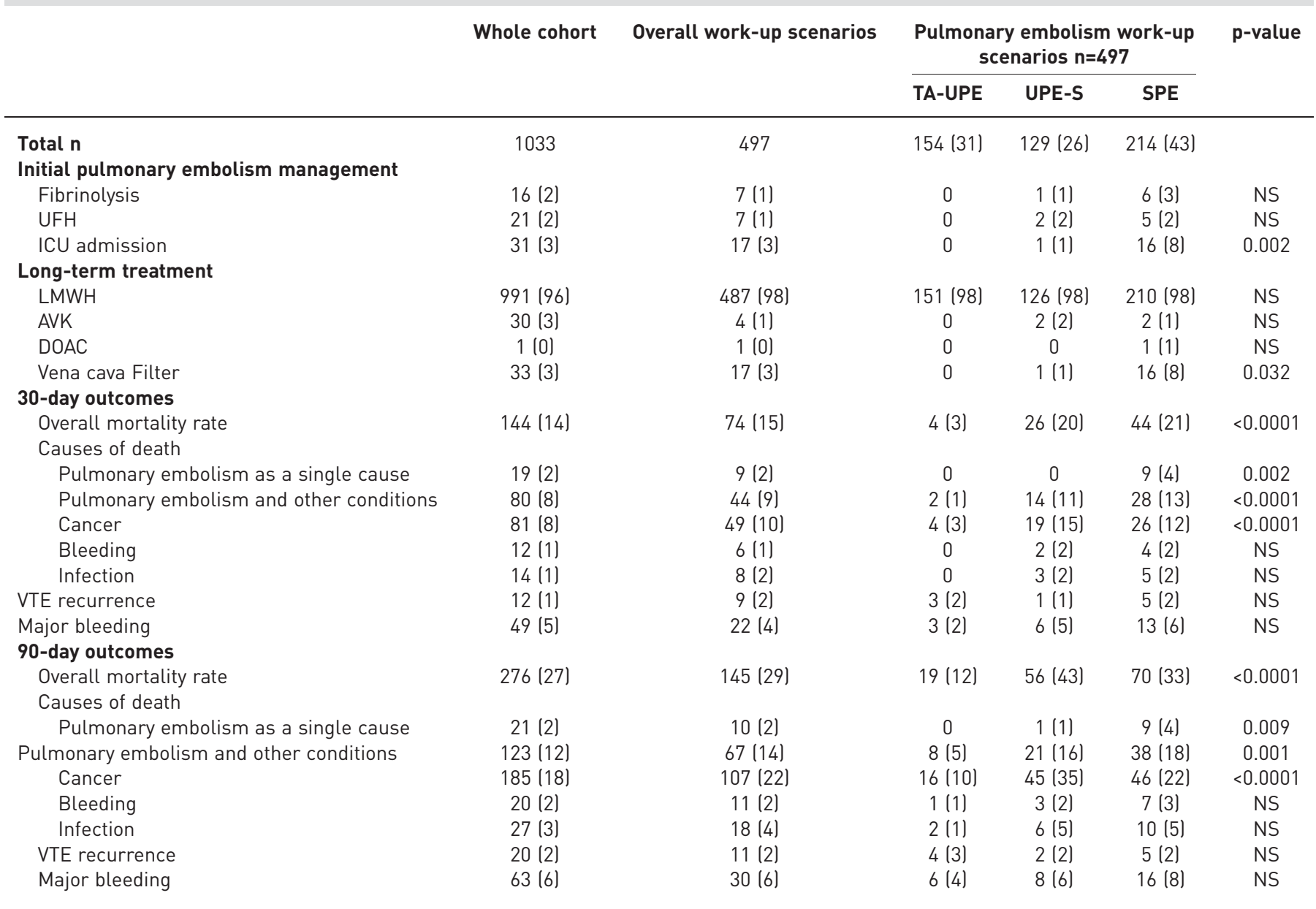

Data are presented as $\mathrm{n}(\%)$, unless otherwise stated. UFH: unfractionated heparin; ICU: intensive care unit; LMWH: low molecular weight heparin; AVK: anti-vitamin K agents; DOAC: direct oral anticoagulants; VTE: venous thromboembolism; PE: pulmonary embolism; SPE: suspected pulmonary embolism; TA-UPE: truly asymptomatic pulmonary embolism; UPE: unsuspected pulmonary embolism with symptoms; NS: not significant.

considered the principal cause of death in all the groups. Figure 2 shows the overall survival curves at the 90-day follow-up according to the work-up scenarios. Interestingly, patients with truly asymptomatic events had a higher 90-day overall survival than those with UPE-S (log-rank <0.001) and patients with SPE (log-rank $\mathrm{p}<0.001)$. Notably, the differences did not reach statistical significance on comparing patients with UPE-S and SPE (log-rank p=0.069).

It was of note that the rate of VTE recurrence and major bleeding at the 30- and 90-day follow-ups were similar in all the work-up scenario groups (table 5).

\section{Discussion}

The present study showed overall, high mortality rates (14\% at 30 and $27 \%$ at 90 days of follow-up) in cancer patients with pulmonary embolism including suspected and unsuspected events. Among the potential predictors of 30-day mortality identified, we would like to point out that those related to abnormal vital signs and low oxygen saturation were particularly robust and consistent with previous studies on SPE in the general population and in patients with cancer [4-8]. In addition, a history of VTE was associated with increased mortality. It was of note that we did not specify a cut-off time for distinguishing "VTE history" (days or weeks before the index pulmonary embolism) from "concomitant deep vein thrombosis" which has also been recognised as having prognostic value in patients with pulmonary embolism [29]. This should be considered when interpreting our results. We also confirmed having metastatic cancer as an independent predictor of short-term mortality as previously reported for SPE [8]. Nonetheless, we consider that having metastatic cancer is a variable with limited utility since most 
FIGURE 2 Overall survival KaplanMeier curves at the 90-day followup according to the work-up scenarios. SPE: symptomatic pulmonary embolism; UPE-S: unsuspected pulmonary embolism with symptoms; TA-UPE: truly asymptomatic unsuspected pulmonary embolism.

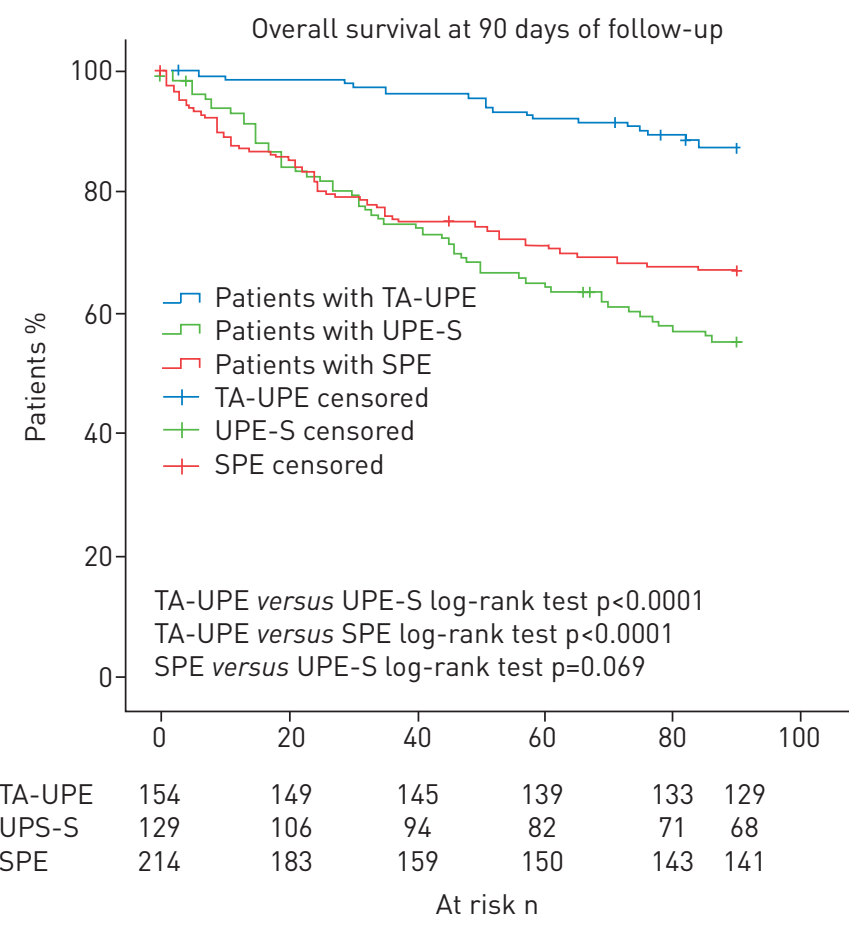

of our patients (75\% in our cohort) had disseminated cancer at pulmonary embolism diagnosis. Our study identified some novel variables which may be potentially useful for predicting the overall 30-day mortality, including: upper gastrointestinal cancers, poor performance status and "cancer progression". Although the variable "cancer progression" would potentially be more discriminative than metastatic disease to predict poor outcomes in this setting, it has the disadvantage of needing specialised cancer-status evaluation which is not always available in emergency departments. Notably, age, chronic lung and heart conditions were not associated with short-term mortality in our study in contrast to previous pulmonary embolism prognostic models for acute SPE in the general population [4-6] and in cancer patients [7, 8]. The more restrictive definition of "active cancer", the inclusion of patients with incidental pulmonary embolism and the great development of anticancer therapies in recent years might explain these remarkable differences between our contemporary cohort and previous studies.

Interestingly, our multiple-choice data collection showed that pulmonary embolism as a single cause of 30-day mortality was reported in only 19 (14\%) out of 144 deaths whereas clinicians mainly attributed mortality to mixed causes. Thus, it seems difficult to decouple the intricate two-way relationship between cancer and thrombosis known to start at a molecular level [30] and discern whether mortality depends on concomitant complications, the thrombotic event itself, or both. These reflections made us rethink the outcome measures that would truly predict the prognosis linked to pulmonary embolism. For instance, a composite endpoint variable including 15-day severe pulmonary embolism-related complications might be of interest. It is also uncertain whether SPE and UPE events should be risk-classified using the same framework or separately.

The present study also confirms the wide clinical spectrum of cancer-associated pulmonary embolism ranging from life-threatening to completely asymptomatic events in both, suspected and unsuspected cases $[31,32]$. Although it has long been known that pulmonary embolism can be asymptomatic [33], broad practical interest concerning this issue has emerged in recent years in parallel to the development and widespread use of imaging tests, particularly in patients with malignancies. Overall, a remarkable $44 \%$ of our prospectively-assessed patients were found to be asymptomatic at pulmonary embolism presentation. In order to categorise the clinical heterogeneity of the cohort, we defined three clinically meaningful work-up scenarios using criteria based on our own daily clinical experience and previous research by O'ConNELL and colleagues $[34,35]$ who described the presence of symptoms as a key prognostic factor in the evaluation of patients with incidental pulmonary embolism. However, in our opinion, the presence of chest symptoms or fatigue may often be misinterpreted by both patients and physicians as secondary to cancer, anti-cancer therapies or other mixed conditions apart from pulmonary embolism itself in this setting. Therefore, we decided to enhance our work-up scenario definitions by adding the setting (in-versus outpatient), clinical pulmonary embolism suspicion (SPE versus UPE) and data on vital signs to the presence of symptoms in order to strengthen the concept of "truly asymptomatic" pulmonary embolism (versus classical acute 
symptomatic pulmonary embolism and the ill-classifiable UPE-S group). A substantial proportion of patients with cancer-related pulmonary embolism (31\% considering the whole group and more than half of those who were unsuspected) fulfilled our easily assessable concept of "truly asymptomatic" pulmonary embolism. This TA-UPE group was identified in our study as having a lower risk of short-term mortality compared to patients with SPE and UPE-S. These findings may help to design specific trials aimed at evaluating optimal acute-phase pulmonary embolism management and broaden full outpatient management in selected low-risk patients with cancer-associated pulmonary embolism [23, 36, 37].

Notably, recurrent VTE and major bleeding rates were similar in all the scenarios suggesting that the underlying prothrombotic proclivity and bleeding risk would be similar irrespective of UPE or SPE at diagnosis taking into account that all our patients were receiving anticoagulation. These findings would, $a$ priori, further support current recommendations on treating UPE and SPE events similarly with anticoagulants. However, from our point of view, the demonstration that the benefits of anticoagulation outweigh the risks as suggested by a recent pooled analysis of 926 patients with UPE [38], as well as optimal dose and duration of this potentially harmful and expensive therapy [39], should be confirmed in properly designed interventional trials particularly in patients with truly asymptomatic events.

Our study has limitations. First, the lower stringency of treatment control compared to controlled trials despite all our patients starting anticoagulation at pulmonary embolism diagnosis. Second, as we included only patients routinely anticoagulated, the study does not allow knowledge as to what the natural history of untreated UPE would be. Third, the interpretation of clinical nuances may differ among clinicians, and minor or uncommon symptoms potentially attributable to pulmonary embolism were not collected in our study. Finally, the lack of systematic information about troponin levels and right ventricular dysfunction for most of our patients does not allow evaluating the performance of these parameters and the European Society of Cardiology proposed risk classification [19] to predict short-term mortality in this setting.

In conclusion, the present study has allowed the identification of novel predictors of 30-day mortality in cancer patients with suspected and unsuspected pulmonary embolism. Amongst patients with unsuspected events, improved outcomes were observed in patients with TA-UPE compared to those with SPE and UPE-S. These findings may help to develop further prospective interventional trials on acute-phase management of pulmonary embolism in this setting.

\section{Acknowledgements}

This work has been partially reported at the following medical meetings: 7th International Conference in Thrombosis and Hemostasis Issues in Cancer (ICTHIC), Bergamo, Italy, May 9-11, 2014; 50th Annual Meeting of the American Society of Clinical Oncology (ASCO), Chicago, IL, USA, May 30-June 3, 2014; European Society for Medical Oncology (ESMO) Congress, Madrid, Spain, September 26-30, 2014; Multinational Association in Supportive Care in Cancer/ International Society in Oral Oncology (MASCC/ISOO) Symposium, Miami, FL, USA, June 26-28, 2014; XIV Congreso Sociedad Española de Oncología Médica (SEOM), Madrid, Spain, October 22-24, 2014; 23rd Biannual International Congress on Thrombosis, Valencia, Spain, 14-17 May, 2014.

\section{References}

1 Sørensen HT, Mellemkjaer L, Olsen JH, et al. Prognosis of cancers associated with venous thromboembolism. N Engl J Med 2000; 343: 1846-1850.

2 Carson JL, Kelley MA, Duff M, et al. The clinical course of pulmonary embolism. N Engl J Med 1992; 326: $1240-1245$.

3 Laporte S, Mismetti P, Décousus H, et al. Clinical predictors for fatal pulmonary embolism in 15,520 patients with venous thromboembolism: findings from the Registro Informatizado de la Enfermedad TromboEmbolica venosa (RIETE) Registry. Circulation 2008; 117: 1711-1716.

4 Wicki J, Perrier A, Perneger TV, et al. Predicting adverse outcome in patients with acute pulmonary embolism: A risk score. Thromb Haemost 2000; 84: 548-552.

5 Donzé J, Le Gal G, Fine MJ, et al. Prospective validation of the pulmonary embolism severity index: a clinical prognostic model for pulmonary embolism. Thromb Haemost 2008; 100: 943-948.

6 Jimenez D, Aujesky D, Moores L, et al. Simplification of the pulmonary embolism severity index for prognostication in patients with acute symptomatic pulmonary embolism. Arch Intern Med 2010; 170: 1383-1389.

7 Kline JA, Roy PM, Than MP, et al. Derivation and validation of a multivariate model to predict mortality from pulmonary embolism with cancer: The POMPE-C tool. Thromb Res 2012; e194-e199.

8 Den Exter PL, Gómez V, Jiménez D, et al. A clinical prognostic model for the identification of low-risk patients with acute symptomatic pulmonary embolism and active cancer. Chest 2013; 143: 138-145.

9 Carmona-Bayonas A, Font C, Jiménez-Fonseca P, et al. On the necessity of new decision-making methods for cancer-associated, symptomatic, pulmonary embolism. Thromb Res 2016; 143: 76-85.

10 Dentali F, Ageno W, Becattini C, et al. Prevalence and clinical history of incidental, asymptomatic pulmonary embolism: a meta-analysis. Thromb Res 2010; 125: 518-522.

11 Sun JM, Kim TS, Lee J, et al. Unsuspected pulmonary emboli in lung cancer patients: the impact on survival and the significance of anticoagulation therapy. Lung Cancer 2010; 69: 330-336.

12 Font C, Farrús B, Vidal L, et al. Incidental versus symptomatic venous thrombosis incancer patients. Analysis of a prospective observational series with 340 consecutive patients. Ann Oncol 2011; 23: 2101-2106. 
13 Shinagare AB, Okajima Y, Oxnard GR, et al. Unsuspected pulmonary embolism inlung cancer patients: comparison of clinical characteristics and outcome with suspected pulmonary embolism. Lung Cancer 2012; 78: $161-166$

14 van Es N, Bleker SM, Di Nisio M. Cancer-associated unsuspected pulmonary embolism. Thromb Res 2014; 133: S172-S178.

15 den Exter PL, Kroft LJ, van del Hulle T, et al. Embolic burden of incidental pulmonary embolism diagnosed on routinely performed contrast-enhanced computed tomography imaging in cancer patients. $J$ Thromb Haemost 2013; 11: 1620-1622.

16 O'Connell C, Razavi P, Ghalichi M, et al. Unsuspected pulmonary emboli adversely impact survival in patients with cancer undergoing routine staging multi-row detector computed tomography scanning. J Thromb Haemost 2011; 9: 305-311.

17 den Exter PL, Hooijer J, Dekkers OM, et al. Risk of recurrent venous thromboembolism and mortality in patients with cancer incidentally diagnosed with pulmonary embolism: a comparison with symptomatic patients. J Clin Oncol 2012; 29: 2405-2409.

18 Kearon C, Akl EA, Omelas J, et al. Antithrombotic therapy for VTE Disease: CHEST Guideline and Expert Panel Report. Chest 2016; 149: 315-352.

19 Konstantinides S, Torbicki A, Agnelli G, et al. 2014 ESC guidelines on the diagnosis and management of acute pulmonary embolism. Eur Heart J 2014; 35: 3033-3069.

20 Di Nisio M, Lee AY, Carrier M, et al. Diagnosis and treatment of incidental venous thromboembolism in cancer patients: guidance from the SSC of the ISTH. J Thromb Haemost 2015; 13: 880-883.

21 Font C, Carmona-Bayonas A, Plasencia JM, et al. Pulmonary embolism in patients with cancer: foundations of the EPIPHANY study. Med Clin (Barc) 2015; 144 Suppl 1: 31-37.

22 Value of the ventilation/perfusion scan in acute pulmonary embolism. Results of the prospective investigation of pulmonary embolism diagnosis (PIOPED). The PIOPED Investigators. JAMA 1990; 263: 2753-2759.

23 Simel DL. Approach to the patient: history and physical examination. In: Goldman L, Schafer AI, eds. Goldman's Cecil Medicine. 24th edn. Saunders Elsevier, Philadelphia, 2011; pp. 22-27.

24 Schulman S, Kearon C. Definition of major bleeding in clinical investigations of antihemostatic medicinal products in non-surgical patients. J Thromb Haemost 2005; 3: 692-694.

25 Font C, Carmona-Bayonas A, Fernández-Martinez A, et al. Outpatient management of pulmonary embolism in cancer: data on a prospective cohort of 138 consecutive patients. J Natl Compr Canc Netw 2014; 12: 365-373.

26 Kuderer NM, Lyman GH. Guidelines for treatment and prevention of venous thromboembolism among patients with cancer. Thromb Res 2014; 133 Suppl: S122-S127.

27 Oken, MM, Creech RH, Tormey DC, et al. Toxicity And response criteria Of The Eastern Cooperative Oncology Group. Am J Clin Oncol 1982; 5: 649-655.

28 Therasse P, Arbuck SG, Eisenhauer EA, et al. New guidelines to evaluate the response to treatment in solid tumors. J Natl Cancer Inst 2000; 92: 205-216.

29 Becattini C, Cohen AT, Agnelli G, et al. Risk stratification of patients with acute symptomatic pulmonary embolism based on presence or absence of lower extremity deep vein thrombosis: systematic review and meta-analysis. Chest 2016; 149: 192-200.

30 Falanga A, Marchetti M, Russo L. The mechanisms of cancer-associated thrombosis. Thromb Res 2015; 135: Suppl 1, S8-S11.

31 Tapson VF. Acute pulmonary embolism. N Engl J Med 2008; 358: 1037-1052.

32 Agnelli G, Becattini C. Acute pulmonary embolism. N Engl J Med 2010; 363: 266-274.

33 Monreal M, Ruiz J, Fraile M, et al. Prospective study on the usefulness of lung scan in patients with deep vein thrombosis of the lower limbs. Thromb Haemost 2001; 85: 771-774.

34 O'Connell CL, Boswell WD, Duddalwar V, et al. Unsuspected pulmonary emboli in cancer patients: clinical correlates and relevance. J Clin Oncol 2006; 24: 4928-4932.

35 O'Connell CL, Razavi PA, Liebman HA. Symptoms adversely impact survival among patients with cancer and unsuspected pulmonary embolism. J Clin Oncol 2011; 29: 4208-4209.

36 Streiff M. Diagnosis and initial treatment of venous thromboembolism in patients with cancer. J Clin Oncol 2009; 27: 4889-4894.

37 Palmer J, Bozas G, Stephens A, et al. Developing a complex intervention for outpatient management of incidentally diagnosed pulmonary embolism in cancerpatients. BMC Health Serv Res 2013; 13: 235.

38 Van der Hulle T, den Exter P, Planquette B, et al. Risk of recurrent venous thromboembolism and major haemorrhage in cancer-associated incidental pulmonary embolism amongst treated and untreated patients: a pooled analysis of 926 patients. J Thromb Haemost 2016; 14: 105-113.

39 Zwicker JI, Bauer KA. How long is long enough? Extended anticoagulation for the treatment of cancer-associated deep vein thrombosis. J Clin Oncol 2014; 32: 3596-3599. 